\title{
CONTRAST ENHANCED CT UNVEILED GIANT RASMUSSEN'S ANEURYSM: A RARE COMPLICATION AND CAUSE OF DEATH IN PULMONARY TUBERCULOSIS
}

\author{
Sanjeev Suman¹, Babita², Shams Tabrez Alam³, G. N. Singh ${ }^{4}$
}

\section{HOW TO CITE THIS ARTICLE:}

Sanjeev Suman, Babita, Shams Tabrez Alam, G. N. Singh. “Contrast Enhanced CT Unveiled Giant Rasmussen's Aneurysm: A Rare Complication and Cause of Death in Pulmonary Tuberculosis". Journal of Evolution of Medical and Dental Sciences 2014; Vol. 3, Issue 18, May 05; Page: 5004-5008,

DOI: $10.14260 /$ jemds/2014/2544

ABSTRACT: Rasmussen's aneurysm is a rare complication of pulmonary tuberculosis. Often the aneurysm ruptures in the bronchial tree causing massive hemoptysis resulting in death. We are reporting a case of partially thrombosed giant Rasmussen's aneurysm which arose in the vicinity of a tuberculous cavity along with empyema necessitans. Unfortunately the patient died of massive hemoptysis within two days of his diagnosis.

KEYWORDS: Giant Rasmussen's, pulmonary tuberculosis.

INTRODUCTION: Rasmussen's aneurysm is a pseudo-aneurysm arising from pulmonary artery which lies in close vicinity of a tuberculosis cavity. In chronic cavitatory pulmonary tuberculosis, mycobacterium tuberculi erode the wall of pulmonary artery causing focal saccular dilatation of the artery (psedoaneurysm) which is referred to as Rasmussen's aneurysm. The source of hemoptysis in cavitary pulmonary tuberculosis is usually from the bronchial arteries. Although pseudoaneurysm of bronchial arteries is an important source of hemoptysis, it is seen infrequently. Pulmonary artery pseudoaneurysms are occasionally encountered in patients with hemoptysis due to pulmonary tuberculosis.

CASE REPORT: A 50 years old female patient was referred from medicine department with the complaints of low grade fever, weight loss, loss of appetite and shortness of breath. Chest $\mathrm{x}$ ray revealed large thick walled cavity in the right middle and lower zone along with patchy consolidation of the upper zone and mild ipsilateral pleural effusion. A band of air density tracking along the intercostal space was observed and seemed to extend into extrathoracic chest wall.(Fig-1) Patient's labs were Total leukocyte count-10900(L-40\%, N-45\%, E- 8\%, B-7\%). High resolution tomography (CT) and contrast enhanced CT scan was planned to help in further management.

High resolution CT and contrast enhanced CT was done on GE 16 slice Multi detector CT machine. Imaging revealed a large thick walled cavity having air filled level in the right middle and lower lobe along with consolidative changes in adjacent lung parenchyma. (Fig. 2 \& 3)The cavity had ruptured into the chest wall through the intercostal space suggesting empyema necessitans. Patchy consolidation with necrotic changes noticed in the posterior segment of upper lobe (Fig. 4 \& 5). Moderate amount of empyema with enhancing pleura was seen. A round intensely enhancing lesion of size $3.2 \times 3.1 \times 3.1 \mathrm{~cm}$ was noticed in the lower lobe lying in the vicinity of lower lobe cavity. Lesion enhancement was similar to the adjacent pulmonary artery along with mild dilatation of lower descending pulmonary artery (Fig 6, 7, 8 \& 9). 


\section{CASE REPORT}

Based on the imaging and clinical features a provisional diagnosis of pulmonary tuberculosis with partially thrombosed pseudoaneurysm of pulmonary artery was made. Pleural tapping was done under CT guidance which confirmed it the tuberculous etiology. Two days after the contrast enhanced CT examination, patient had three bouts of massive hemoptysis and unfortunately died of asphyxia and hypovolemic shock.

\section{IMAGING FEATURES:}

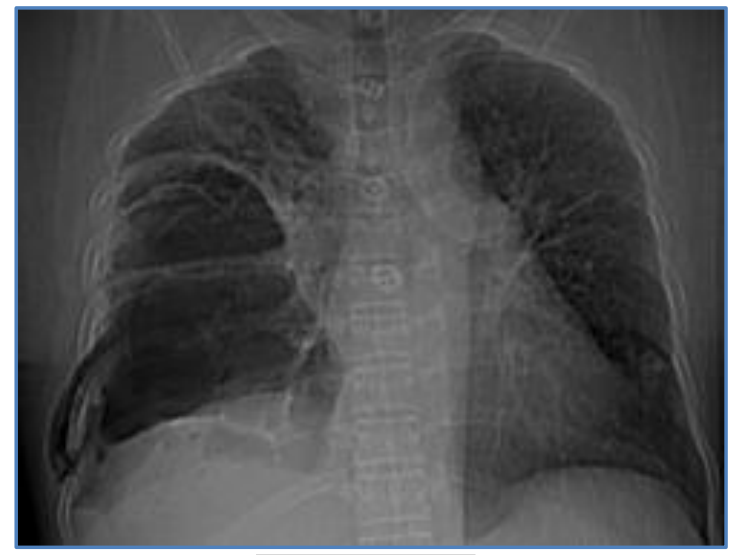

\section{Figure 1}

Figure.1: Chest $\mathrm{x}$ ray PA view showing Large thick walled cavity in the right mid and lower zone region along with patchy consolidation in the upper zone and mild ipsilateral pleural effusion. A band of air density tracking along the intercostal space extending in to extrathoracic chest wall.

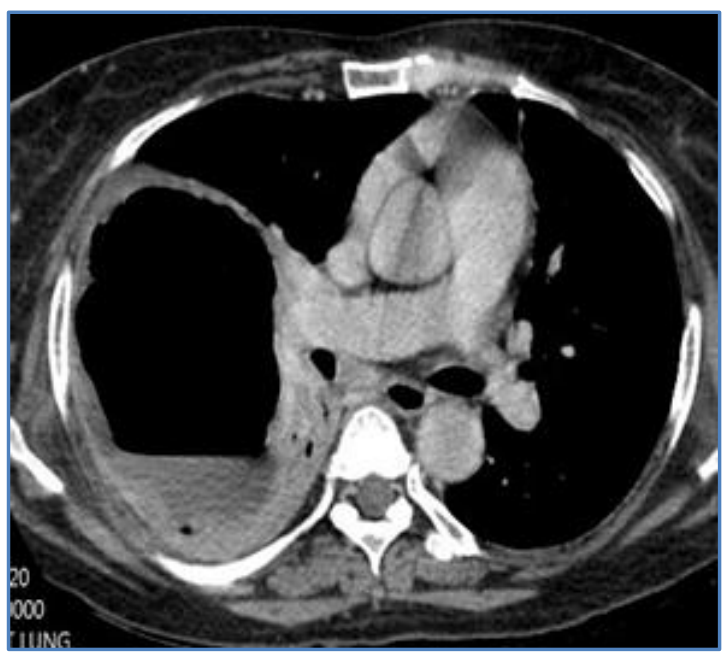

Figure 2

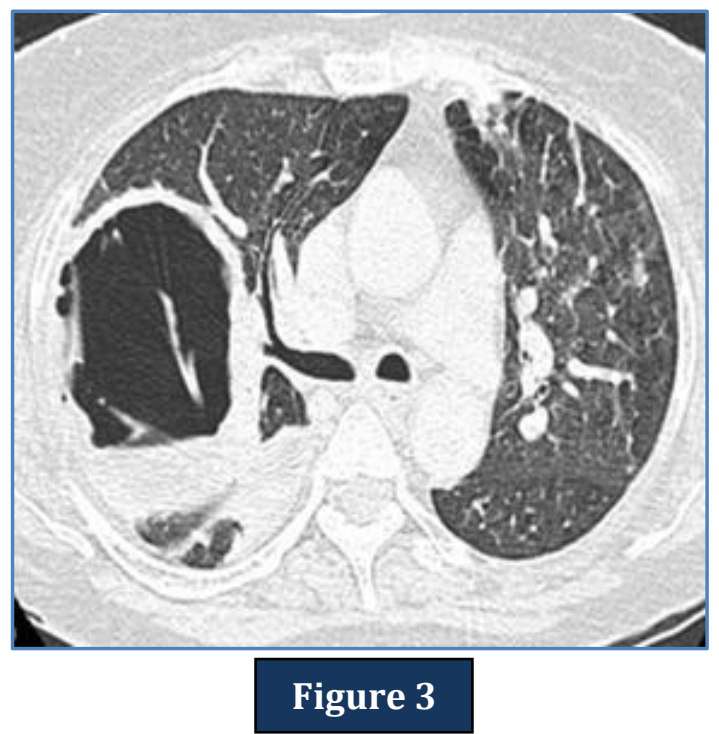

Figure 3

Figure 2 \& 3: Axial CECT \& HRCT images showing large thick walled cavity with air fluid level and ground glass opacity in the left perihilar region. 


\section{CASE REPORT}

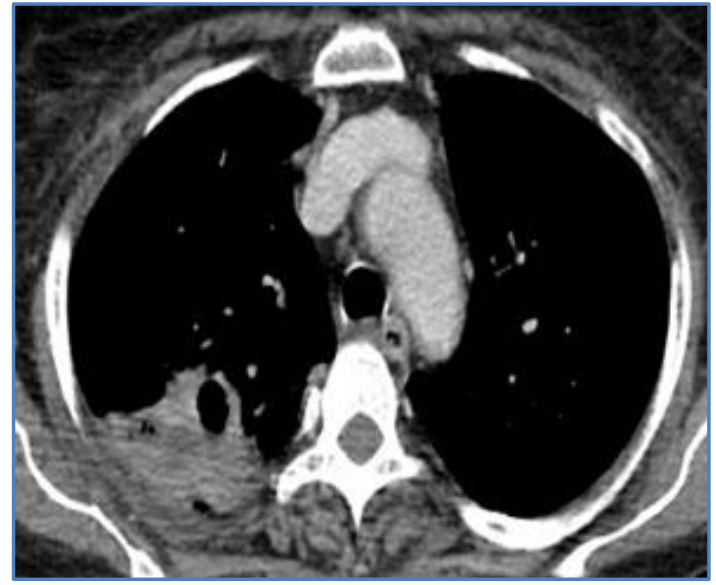

Figure 4

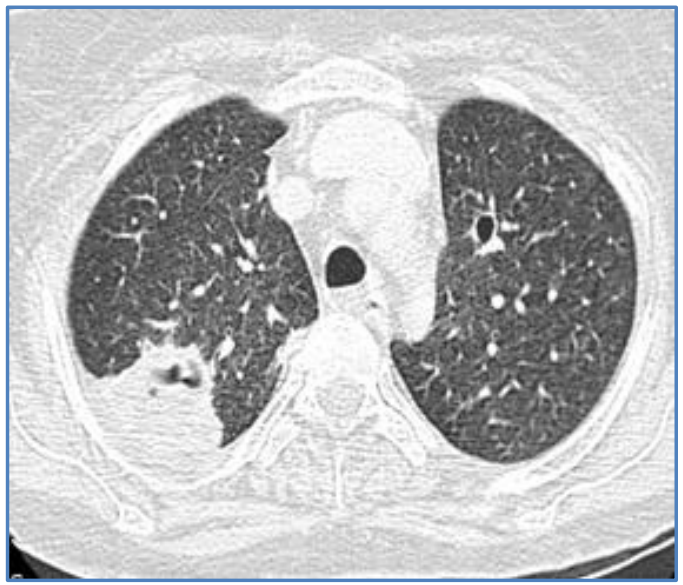

Figure 5

Figure 4 \& 5: CECT \& HRCT images showing patchy consolidation in the posterior segment of right upper lobe.

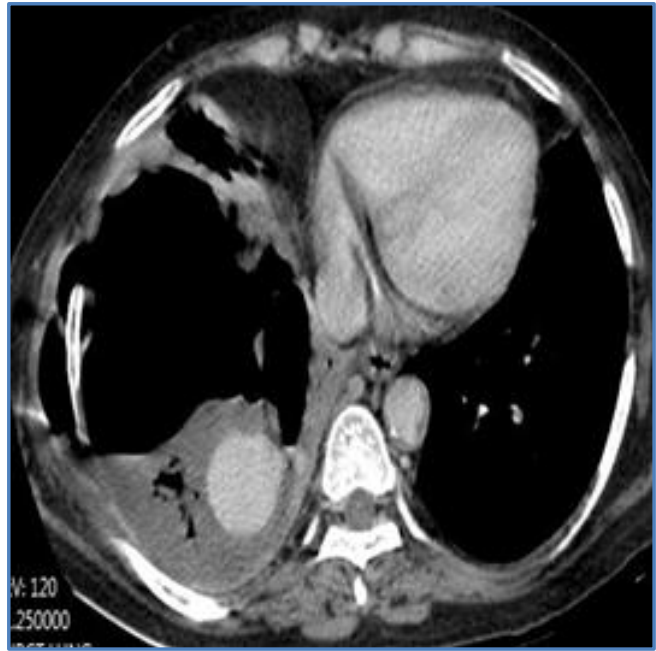

Figure 6

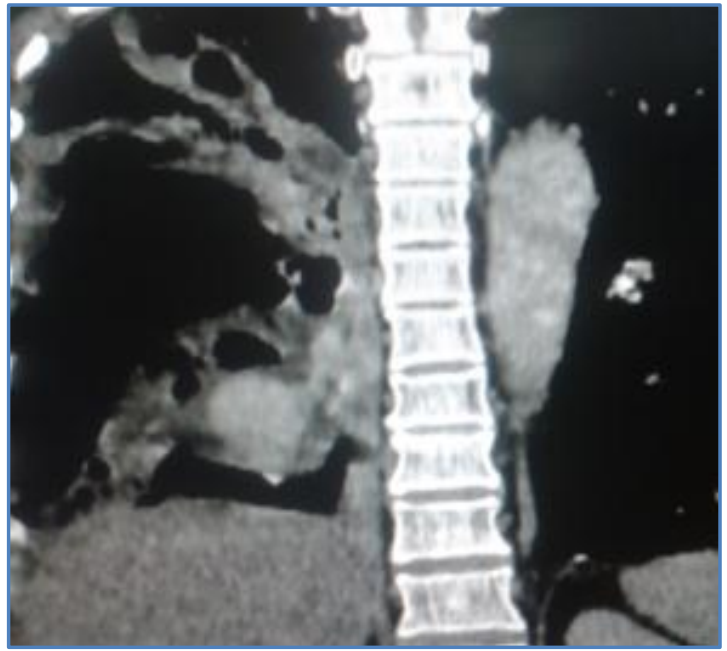

Figure 7

Figure 6 \& 7: Axial CECT and coronal reformatted images showing a large partially thrombosed pseudoaneurysm arising from right descending pulmonary artery, which appear dilated. 


\section{CASE REPORT}

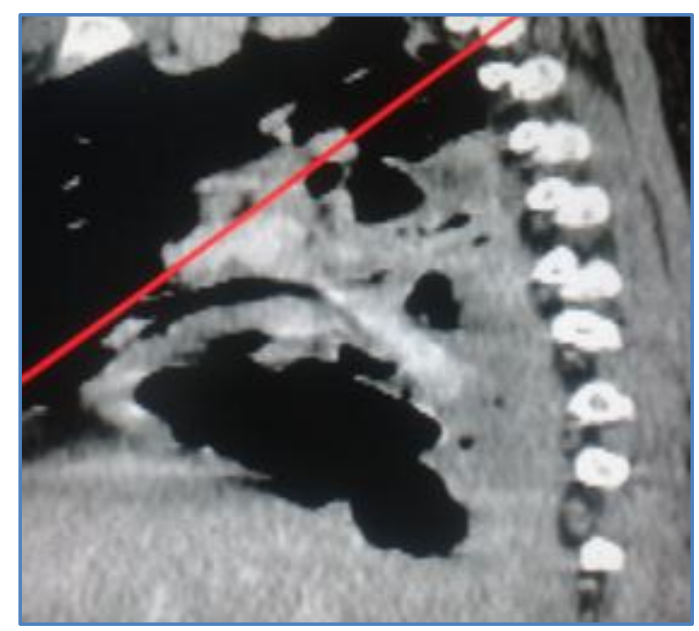

Figure 8

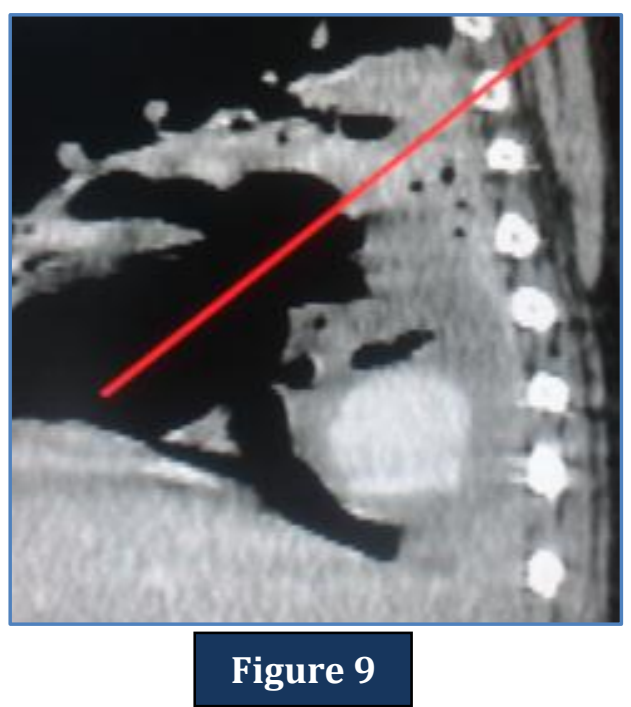

Figure 9

Figure 8 \& 9: Sagittal reformatted images showing partially thrombosed pseudoaneurysm of pulmonary artery with dilated descending pulmonary artery.

DISCUSSION: Fritz Valdemar Rasmussen, a Danish physician, who first described the rasmussen's aneurysm as a pulmonary vessel passing through the wall of a tuberculous pulmonary cavity with an aneurysmal dilatation of this vessel into the cavity. ${ }^{1}$ There are many complication and sequelae of pulmonary tuberculosis including bronchiectasis, pulmonary fibrosis, aspergilloma (in the cavity of post primary pulmonary tuberculosis), broncholithiasis, empyema, hypertrophy of the bronchial artery and Rasmussen's aneurysm. Out of all the complications, pulmonary vascular aneurysm or pseudoaneurysm has devastating course and required urgent and aggressive intervention; otherwise patient can die of massive hemoptysis. ${ }^{2}$

A review of autopsy findings in patients with a history of chronic cavitary tuberculosis showed a 5\% prevalence of Rasmussen's aneurysm ${ }^{3}$. However a study have found that almost $11 \%$ of patients undergoing bronchial angiography for hemoptysis had visible pulmonary artery pseudoaneurysms, and in the subset of patients with chronic pulmonary tuberculosis, the incidence was $14 \%{ }^{4}$ Hemoptysis is the usual presenting symptom and may be life-threatening when it is massive. Progressive weakening of the arterial wall occurs as granulation tissue replaces both the adventitia and the media. The granulation tissue in the vessel wall is then gradually replaced by fibrin, resulting in thinning of the arterial wall, pseudoaneurysm formation, and subsequent rupture..$^{3,4}$

The etiology of Solitary peripheral pulmonary artery aneurysms includes trauma, infection, congenital or acquired pulmonary vascular abnormalities and pulmonary hypertension. A destructive pathology in the lung irrespective of the etiology erodes the adjacent structures in the lung. The eponym Rasmussen's aneurysm refers specifically to tuberculous etiology. They are usually peripheral and beyond the branches of main pulmonary artery. ${ }^{5}$ These pseudoaneurysms are uncommon and may form months to years after formation of the cavity. ${ }^{2}$ 
CONCLUSION: A Rasmussen's aneurysm is a rare life threatening complication of cavitary tuberculosis caused by granulomatous weakening of a pulmonary arterial wall. Assessment with contrast-enhanced CT allows accurate evaluation of pulmonary artery aneurysms and pseudoaneurysms, facilitating prompt diagnosis and treatment. Every suspected cavitary tuberculosis patient should be assessed with contrast enhanced CT of thorax, for the early detection and timely intervention of pulmonary or bronchial artery aneurysm.

\section{REFERENCES:}

1. Rasmussen V. On haemoptysis, especially when fatal, in its anatomical and clinical aspects. Edinburgh Med J 1868; 14:385-401.

2. Auerbach 0. Pathology and pathogenesis of pulmonary arterial aneurysm in tuberculous cavities. Am Rev Tuber 1939; 39:99-115.

3. Santelli ED, Katz DS, Goldschmidt AM, Thomas HA. Embolization of multiple Rasmussen aneurysms as a treatment of hemoptysis. Radiology 1994; 193:396-398.

4. Remy J, Smith M, Lemaitre L, Marache P, Fournier E. Treatment of massive hemoptysis by occlusion of a Rasmussen aneurysm. Am J Roentgenol 1980; 135:605-606.

5. Hala Sbano, Adam W Mitchell, Philip W Ind, James E Jackson. Peripheral Pulmonary Artery Pseudoaneurysms and Massive Hemoptysis. American Journal of Roentgenology. 2005; 184: 1253-1259.

\section{AUTHORS:}

1. Sanjeev Suman

2. Babita

3. Shams Tabrez Alam

4. G. N. Singh

\section{PARTICULARS OF CONTRIBUTORS:}

1. Senior Resident, Department of Radiodiagnosis, Patna Medical College and Hospital.

2. Tutor, Department of Microbiology, Patna Medical College and Hospital.

3. Post Graduate, Department of Radiodiagnosis, Patna Medical College and Hospital.

4. Professor and HOD, Department of Radiodiagnosis, Patna Medical College and Hospital.

\author{
NAME ADDRESS EMAIL ID OF THE \\ CORRESPONDING AUTHOR: \\ Dr. Sanjeev Suman, \\ Senior Resident, \\ Department of Radiodiagnosis, \\ Patna Medical College and Hospital, \\ Nalanda Scan Center, \\ \#0/63, Doctor's Colony, \\ Kankarbagh, Patna- 800020, Bihar. \\ E-mail: drbabitasmn@gmail.com \\ Date of Submission: 31/03/2014. \\ Date of Peer Review: 01/04/2014. \\ Date of Acceptance: 22/04/2014. \\ Date of Publishing: 05/05/2014.
}

